\title{
The Evaluation Transformer Model of Reduction and Its Correctness
}

\author{
G L Burn \\ Department of Computing, Imperial College, 180 Queen's Gate, \\ London SW7 2BZ, United Kingdom
}

\begin{abstract}
Lazy evaluation of functional programs incurs time and memory overheads, and restricts parallelism compared with programs that are evaluated strictly. A number of analysis techniques, such as abstract interpretation and projection analysis, have been developed to find out information that can alleviate these overheads. This paper formalises an evaluation model, the evaluation transformer model of reduction, which can use information from these analysis techniques, and proves that the resulting reduction strategies produce the same answers as those obtained using lazy evaluation.
\end{abstract}

\section{Introduction}

Popular mythology about implementations of lazy functional languages is that they are slow when compared with more traditional languages such as $\mathrm{C}$ and Pascal. Early implementations were slow, for two reasons:

- they were largely interpretive; and

- the semantics of such languages requires that arguments to functions are not evaluated until their values are needed, imposing time and memory overheads, and restricting any parallelism in an implementation.

Compiler technology has advanced sufficiently so that lazy functional programs now run respectably fast when compared with those written in more traditional languages, overcoming the overheads due to interpretation.

This paper tackles the second problem. Specifically we:

- describe a more efficient implementation model, the evaluation transformer model, that can be used when information is known about how functions use their arguments; and

- describe a semantically sound analysis technique, abstract interpretation, which can determine this information.

Evaluation transformer information can be used to generate more efficient code for sequential and parallel machines.

In more detail, the story-line of the paper is as follows. 
Lazy evaluation is restrictive evaluation mechanism in two ways. Firstly, it only ever knows the next step that must be performed in executing a program. Secondly, it only ever evaluates expressions to head normal form ${ }^{1}$.

The evaluation transformer model of reduction lifts both of these restrictions. It introduces the concept of an evaluator, which specifies the amount of evaluation to do to an expression. With each argument to a function, we associate an evaluation transformer, which says how much evaluation of that argument expression can be done, given that a certain amount of evaluation is allowed of the function application. Using this information, we may know that there are several expressions which must be evaluated, and that they need more evaluation than to head normal form.

Functional languages can be studied from both operational and denotational viewpoints. The operational viewpoint tells us how they can be implemented, and the freedom we have in the implementation, whilst the denotational viewpoint gives the meanings of programs in terms of mathematical structures called domains.

The key point is that we want to use denotational information about a program in order to implement the prograrn more efficiently, that is, change its operational behaviour.

There is an important relationship between the operational and denotational viewpoints, which must be preserved in any implementation model.

We ensure that the evaluation transformer model of reduction preserves this relationship by using a semantically sound analysis technique, called abstract interpretation, to determine evaluation transformers.

Our theoretical development is done in terms of the typed lambda calculus (typed $\lambda$ calculus); it allows us to study the properties of functional languages without worrying about the concrete syntax of a particular language.

The main contributions of this paper are a formalisation of evaluators as reduction strategies in the typed lambda calculus, and a proof that using the evaluation transformers obtained from an abstract interpretation results in a safe reduction strategy. By safe, we mean that the evaluation of a program will terminate with a head normal form of the program if and only if one exists, that is, produce the same answer as lazy evaluation. Evaluation transformers were first discussed in [Bur87a, Bur87b], and the abstract interpretation framework we use in [Bur87b].

As well as presenting these results, this paper presents the foundations on which they are built, giving a complete overview of the work. In summary, the paper covers:

- an informal introduction to the evaluation transformer model (Section 2);

- the definition of the language $\Lambda_{T}$, a typed lambda calculus with constants. This language has enough data types and constants to illustrate the main ideas of the paper (Section 3);

\footnotetext{
${ }^{1}$ We realise that most implementations in fact do some form of combinator reduction to weak head normal form (defined in [Pey87, p. 198]). However, the theory of such a reduction system is not as wellfounded at present as that of reduction to head normal form, although work on the lazy lambda calculus looks promising [Abr90, Ong88b, Ong88a], and so we prefer to work instead with reduction to head normal form. In passing, we note a tendency of the implementation community to quote results about $\beta$-reduction of an untyped lambda-calculus without constants in order to justify their implementations of typed languages with constants, based on combinator reduction to weak head normal form. This paper attempts to be somewhat more rigorous.
} 
- the execution of programs written in $\Lambda_{T}$ is formalised using $\beta$ - and $\delta$-reduction. Two important theorems are presented: (i) the Church-Rosser Theorem, which gives the freedom we have in varying the implementation; and (ii) the Head Normalisation Theorem, which states that the leftmost reduction strategy is guaranteed to terminate with an answer from a program if it has one (Section 4);

- the standard interpretation of $\Lambda_{T}$, which is its usual denotational semantics (Section 5);

- the standard interpretation of a program is bottom if and only if the leftmost reduction strategy fails to terminate. This is the crucial theorem in relating the operational and denotational semantics of $\Lambda_{T}$, and is an extension of the result for PCF [Plo77] (Section 6);

- evaluators are formalised as reduction strategies associated with particular Scottclosed sets; the evaluation of an expression with a particular evaluator fails to terminate if and only if the standard interpretation of the expression is in the Scott-closed set associated with the evaluator. Given information about the behaviour of functions over Scott-closed sets, evaluation transformers can be determined which result in safe evaluation strategies (Section 7); and

- we show how the behaviour of functions over Scott-closed sets can be determined using abstract interpretation, and give a particular abstract interpretation which can be used to determine the evaluation transformers informally motivated in Section 2 (Section 8).

Finally, we draw the results together in Section 9, and indicate further work that must be done.

The use of evaluation transformers in compiling code for sequential and parallel machines is extensively discussed in [Bur90, Bur91b].

The results of this paper are discussed at much greater length in a book by the author [Bur91b].

\section{The Evaluation Transformer Model of Reduction}

\subsection{Evaluators}

We know that in some function applications, the argument will need more reduction than just to head normal form (HNF). Suppose that the data type list has been declared as ${ }^{2}$ :

$$
\text { list } *::=\mathrm{Nil} \mid \text { Cons * (list *) }
$$

and the function length defined by:

\footnotetext{
${ }^{2}$ All example code fragments will be written in Miranda [Tur86]. Miranda is a trademark of Research Software Ltd.
} 


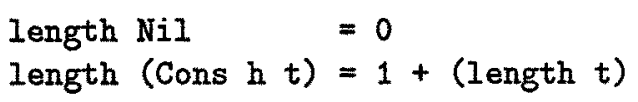

To evaluate an application of length, the whole of the structure of the argument list will need to be traversed, but the values which are the first argument to the constructor Cons will never have to be evaluated. The function sum however, also has to evaluate the first argument to the constructor Cons for each element in the list.

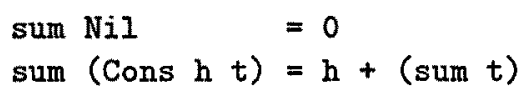

We will call the process of recursively evaluating the expressions which are in the recursive places in a data type definition, creating the structure of the expression. The process will only terminate if the structure of the object is finite.

We will say that we can evaluate an expression using a particular evaluator, and call an evaluator which evaluates expressions to $\mathrm{HNF} \xi_{H N F}$, an evaluator which evaluates the structure of a data type $\xi_{T}$ (the $T$ reminding us that all the tails have to be evaluated), and an evaluator which evaluates the structure of a data type and every non-recursive element of the data type to HNF $\xi_{T H}$ (reminding us that all the heads and tails have to be evaluated). For completeness, the evaluator $\xi_{N O}$ does no evaluation. There is an obvious generalisation of the concept of evaluators to the situation where more evaluation is done to substructures than that done by $\xi_{T H}$, and to other recursively defined data types.

Note that although $\xi_{T}$ and $\xi_{T H}$ are defined in terms of evaluating the whole structure of an expression, they could be implemented so that they only evaluated a certain number of elements of the data structure at a time; when the evaluated part of the list has been consumed, the evaluation of the expression could be reawakened to evaluate some more.

\subsection{Evaluation Transformers}

Not only do some functions require more evaluation of their argument than to HNF, but the amount of evaluation of an argument may depend on the amount of evaluation required of the application. Consider for example the function append defined by

$$
\begin{aligned}
\text { append Nil ys } & =y s \\
\text { append (Cons } x \text { xs) ys } & =\text { Cons } \mathrm{x} \text { (append } \mathrm{xs} \text { ys) }
\end{aligned}
$$

Normally only the first argument to append needs to be evaluated to HNF because a result in HNF is required. However, if one was to require that an application of append was to deliver the structure of a list, such as in the application length (append e1 e2), then clearly it can only do this if the structure of both of its arguments are created.

For each argument of a function therefore, we have to determine an evaluation transformer. An evaluation transformer for an argument is a function which says which evaluator may be used for evaluating the argument, given the evaluator for the expression. For example, the evaluation transformers for append are given in Figure 1. APPENDi is the evaluation transformer for the $i$ th argument of append.

If an application of append is being evaluated with the evaluator $\xi_{T}$, then the first argument can be evaluated with the evaluator $A P P E N D_{1}\left(\xi_{T}\right)$ which, from the figure, is 


\begin{tabular}{|c|c|c|}
\hline$E$ & $A P P E N D_{1}(E)$ & $A P P E N D_{2}(E)$ \\
\hline \hline$\xi_{N O}$ & $\xi_{N O}$ & $\xi_{N O}$ \\
\hline$\xi_{H N F}$ & $\xi_{H N F}$ & $\xi_{N O}$ \\
\hline$\xi_{T}$ & $\xi_{T}$ & $\xi_{T}$ \\
\hline$\xi_{T H}$ & $\xi_{T H}$ & $\xi_{T H}$ \\
\hline
\end{tabular}

Figure 1: Evaluation Transformers for append

$\xi_{T}$. Similarly, the second argument can be evaluated with $\xi_{T}$. Further examples can be found in [Bur87b, Bur91b].

\subsection{The Evaluation Transformer Model}

Evaluation transformers say how much evaluation can be done to an argument expression in a function application, given the amount of evaluation that can be done to the application. Knowing that an argument expression needs to be be evaluated means that we can change the evaluation order to make an implementation more efficient. In a sequential machine this means that argument expressions can be evaluated, rather than creating closures for them, whilst in a parallel machine concurrent tasks can be spawned to evaluate them. Knowing that more evaluation than to HNF needs to be done means that further optimisations may be possible, evaluating the whole of a list without building any closures for example.

\section{The Language $\Lambda_{T}-$ A Typed Lambda-Calculus with Constants}

The language $\Lambda_{T}$ is a minimal typed lambda calculus with constants, which will enable us to discuss the main issues of this paper. Its definition can be found in Figures 2 and $3^{3}$. A fuller language, into which most typed lazy functional programs can be translated, is treated in [Bur91b].

The constant lcase (list $\tau) \rightarrow \sigma \rightarrow(\tau \rightarrow($ list $\tau) \rightarrow \sigma) \rightarrow \sigma$ is used in the translation of pattern matching [FH88, Pey87, Rea89].

\section{The Operational Semantics of $\Lambda_{T}$}

In a typed $\lambda$-calculus with constants, program execution is formalised by the notion of reduction. The first type of reduction, called $\beta$-reduction, is when a $\lambda$-abstraction is

\footnotetext{
${ }^{3}$ Strictly speaking, the $\delta$-rules for $\Lambda_{T}$ are also part of its definition. These can be found in Figure 4.
} 
The set $T$ of types is the least set defined by:

$\{$ bool, int $\} \subseteq T$

$\sigma, \tau \in T \Rightarrow(\sigma \rightarrow \tau) \in T$

$\sigma \in T \Rightarrow($ list $\sigma) \in T$

The type system of $\Lambda_{T}$

(1) $x^{\sigma}: \sigma$

(2) $c_{\sigma}: \sigma$

(3) $\frac{e_{1}: \sigma \rightarrow \tau, e_{2}: \sigma}{\left(e_{1} e_{2}\right): \tau}$

(4) $\frac{e: \tau}{\left(\lambda x^{\sigma} . e\right): \sigma \rightarrow \tau}$

(5) $\frac{e: \sigma \rightarrow \sigma}{\left(\operatorname{fix}_{(\sigma \rightarrow \sigma) \rightarrow \sigma} e: \sigma\right)}$

The syntax of $\Lambda_{T}$

Figure 2: The Language $\Lambda_{T}-\mathrm{A}$ Typed Lambda Calculus with Constants

$$
\begin{aligned}
& \text { true }_{\text {bool }} \\
& \text { if }_{\text {bool } \rightarrow \sigma \rightarrow \sigma \rightarrow \sigma} \\
& \left\{\mathbf{0}_{\text {int }}, \mathbf{1}_{\text {int }}, \mathbf{2}_{\text {int }}, \ldots\right\} \\
& \text { +int } \rightarrow \text { int } \rightarrow \text { int }_{\text {bool }} \\
& \text { nil }_{(\text {list } \tau)} \\
& \text { lcase }_{(\text {list } \tau) \rightarrow \sigma \rightarrow(\tau \rightarrow(\text { list } \tau) \rightarrow \sigma) \rightarrow \sigma} \quad \text { cons }_{\tau \rightarrow(\text { list } \tau) \rightarrow(\text { list } \tau)}
\end{aligned}
$$

Figure 3: The Constants Used In $\Lambda_{T}$ 


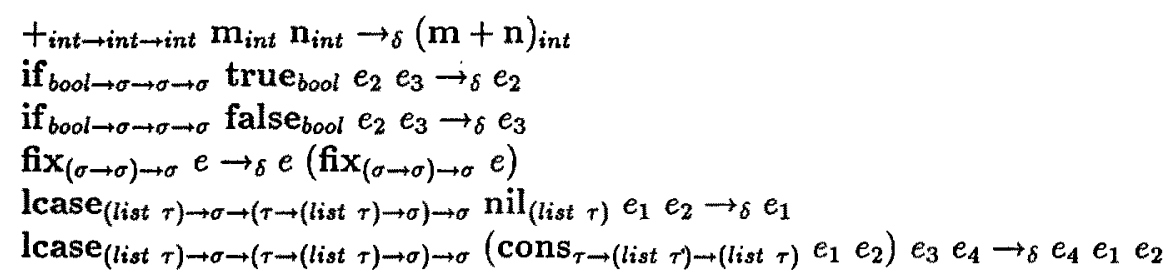

Figure 4: $\delta$-rule Schemata for Constants in $\Lambda_{T}$

applied to a term:

$$
\left(\lambda x^{\sigma} . e_{1}\right) e_{2}
$$

and the result of the reduction step is obtained by substituting $e_{2}$ for all the free occurrences of $x^{\sigma}$ in $e_{1}$. Those not familiar with $\beta$-reduction can find it defined in any text on the $\lambda$-calculus, [Bar84, p. 51] for example.

The term being reduced is called a redex.

In a $\lambda$-calculus with constants, we need to extend the notion of reduction to include applications of functional constants. For example, we would expect that the application:

$$
+_{\text {int } \rightarrow \text { int } \rightarrow \text { int }} 2_{\text {int }} 3_{\text {int }}
$$

should be reduced to $\boldsymbol{5}_{\text {int }}$. Such rules are often called delta-rules (often written $\delta$-rules), and we will say that $e$ one-step $\delta$-reduces to $e^{\prime}$, written $e \rightarrow_{\delta} e^{\prime}$, if $e^{\prime}$ is obtained from $e$ by replacing one occurrence of $e_{1}$ in $e$ with an occurrence of $e_{2}$, where $e_{2}$ is obtained from $e_{1}$ by using one $\delta$-rule. Schemata for the $\delta$-rules for the constants in $\Lambda_{T}$ are given in Figure 4. It should be noted that the $\delta$-rules are an integral part of the definition of the language $\Lambda_{T}$; by changing the $\delta$-rules we change the operational behaviour of programs.

As an aside, we note that fix $x_{(\sigma \rightarrow \sigma) \rightarrow \sigma}$ is used to encode recursion, and is the fixed point combinator. Using fix $x_{(\sigma \rightarrow \sigma) \rightarrow \sigma}$ allows programs admitting infinite reduction sequences to be written in the language $\Lambda_{T}$ (try reducing the expression $\left(f x_{(\sigma \rightarrow \sigma) \rightarrow \sigma}\left(\lambda x^{\sigma} . x\right)\right.$ ) for example).

We will say e one-step beta-delta-reduces (one-step $\beta \delta$-reduces) to $e^{\prime}$, and write $e \rightarrow_{\beta \delta}$ $e^{\prime}$, if either $e \rightarrow_{\beta} e^{\prime}$ or $e \rightarrow_{\delta} e^{\prime}$. It is normal to say that $e \beta \delta$-reduces to $e^{\prime}$ if $e^{\prime}$ results from $e$ by using zero or more one-step $\beta \delta$-reduction steps, and write it:

$$
e \rightarrow \beta \delta e^{\prime}
$$

A term may have more than one redex in it, and so we must ask if the order of reducing redexes matters. We will say that $\beta \delta$-reduction has the Church-Rosser Property for $\Lambda_{T}$ if whenever $e \rightarrow \beta \delta e_{1}$ and $e \rightarrow \beta \delta e_{2}$, then there exists an $e_{3}$ such that $e_{1} \rightarrow_{\beta \delta} e_{3}$ and $e_{2} \rightarrow \beta \delta e_{3}$. 


\section{Theorem 4.1 (Church-Rosser Theorem)}

For the language $\Lambda_{T}$ and the $\delta$-rules given in Figure 4, the notion of reduction $\rightarrow \beta \delta$ has the Church-Rosser property.

\section{Proof}

The proof of this theorem follows from a theorem due to Mitschke [Mit76] (cited as Theorem 15.3.3 of [Bar84]) which gives sufficient conditions for a typed $\lambda$-calculus with constants to be Church-Rosser. See [Bur91b, Theorem 2.1.11].

The practical importance of this theorem for implementations of lazy functional languages is that its says that the order of performing reduction steps does not matter for any terminating reduction sequence, for the results of two such sequences can be reconciled by reducing them to a common third term.

Reduction of term $e$ terminates when an expression in head normal form (HNF) has been reached. A term is in HNF if it is of the form:

- $\lambda x_{1}^{\sigma_{1}} \ldots \lambda x_{n}^{\sigma_{n}} . v e_{1} \ldots e_{k}, k \geq 0, n \geq 0$

where:

1. $v$ is a variable; or

2. $v$ is a constant $c_{\sigma}$ and either

(a) all $\delta$-rules for $c_{\sigma}$ require more than $k$ arguments; or

(b) there are no $\delta$-rules for $c_{\sigma}$.

The Church-Rosser Theorem only gives us information about terminating reduction sequences. We need to know if there is some way of choosing redexes so that a head normal form for a term can be found, if one exists. $\mathrm{HNF}$,

A one-step reduction strategy for $\Lambda_{T}$ is a map, $F: \Lambda_{T} \rightarrow \Lambda_{T}$, if for all $e \in \Lambda_{T}$ not in

$$
e \rightarrow \beta \delta F(e) \text {. }
$$

A reduction strategy is a map $F: \Lambda_{T} \rightarrow \Lambda_{T}$, if for all $e \in \Lambda$,

$$
e \rightarrow \beta \delta F(e) \text {. }
$$

In words, a one-step reduction strategy is a way of choosing the next redex to reduce at each stage of a reduction sequence. The condition that $e \rightarrow_{\beta} F(e)$ means that $e$ must actually reduce to the term $F(e)$ in one step, that is, $F(e)$ must be obtained from $e$ by reducing one redex. Because $F$ is a mapping, for each $e \in \Lambda$ not in $\mathrm{HNF}$, there is a unique redex it chooses. Clearly we will only be interested in computable reduction strategies, that is, given some term $e$ in $\Lambda, F(e)$ can be computed.

If $e_{1}$ and $e_{2}$ are two subterms of some term $e$, then we will say that $e_{1}$ is to the left of $e_{2}$ if either $e_{1}$ occurs entirely to the left of $e_{2}$ in $e$, or $e_{2}$ is a proper subterm of $e_{1}$. For example, in the expression:

$$
+_{i n t \rightarrow i n t \rightarrow i n t}\left(+_{i n t \rightarrow i n t-i n t} 0_{i n t} \mathbf{1}_{i n t}\right) \mathbf{5}_{i n t}
$$


( $\left.+_{\text {int } \rightarrow \text { int } \rightarrow \text { int }} \mathbf{0}_{\text {int }} \mathbf{1}_{\text {int }}\right)$ is to the left of $\mathbf{0}_{i n t}$, and $\mathbf{0}_{i n t}$ is to the left of $\mathbf{5}_{\text {int }}$. "To the left of" is a non-reflexive linear ordering on the occurrences of subterms in $e$. If terms in $\Lambda_{T}$ were fully bracketed, then a subterm $e_{1}$ is to the left of $e_{2}$ if its leftrnost bracket is to the left of the leftmost bracket of $e_{2}$. The leftmost redex in a term $e$ is a subterm of $e$ that is a redex and is to the left of any other subterm of $e$ which is also a redex.

The one-step reduction strategy $F_{\ell}$ is defined by

$$
\begin{aligned}
F_{\ell}(e) & =e \text { if } e \text { is an } \mathrm{HNF} \\
& =e^{\prime} \text { if } e \rightarrow \beta \delta e^{\prime} \text { by reducing the leftmost redex }
\end{aligned}
$$

\section{Theorem 4.2 (Head-Normalisation Theorem)}

For $\Lambda_{T}$ with $\beta \delta$-reduction, $F_{\ell}$ is guaranteed to terminate with a HNF of a term if one exists.

\section{Proof}

The proof of this theorem is quite difficult, and relies on an adaptation of some results due to Hindley [Hin78]. See [Bur91b, Theorem 2.1.14].

The practical importance of this theorem is that it gives an algorithm for choosing the next redex that must be reduced in order to obtain an answer from a program after a finite number of reduction steps, if one exists. Unfortunately, it is restrictive in that it only allows the leftmost redex to be reduced at each step. If we can guarantee that a change in the reduction strategy retains the termination properties of a program, then the Church-Rosser Theorem tells us that the resulting answer from the program in some sense equivalent to the one obtained using the reduction strategy $F_{\ell}$.

\section{The Standard Interpretation of $\Lambda_{T}$}

Any formal language can be given a semantics by giving an interpretation to each of the symbols of the language, and defining how these meanings are to be combined. Usually only one such denotational semantics is given to a language; for $\Lambda_{T}$ we will say that this is its standard interpretation. Later we will give a parameterised abslract interpretation for $\Lambda_{T}$.

An interpretation for $\Lambda_{T}$ consists of two parts:

- a domain as the interpretation of each type; and

- interpretations of the language constructs.

We denote the standard interpretation of the type $\sigma$ by $\mathbf{S}_{\sigma}$, and of the expression $e$ by $\mathbf{S} \llbracket e \rrbracket \rho^{\mathbf{S}}$, with the $\mathbf{S}$ reminding us that it is the standard interpretation. The standard interpretation of the types bool and int are given in Figure 5. In Figure 6, the standard 


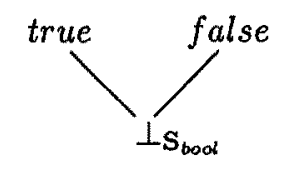

Standard Interpretation of bool

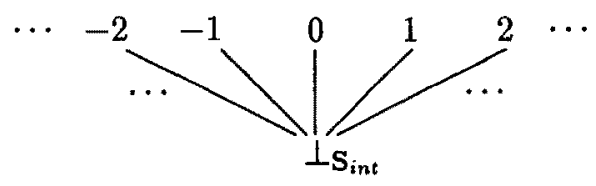

Standard Interpretation of int

Figure 5: The Standard Interpretation of the Types bool and int

interpretation of $\sigma \rightarrow \tau$ is given as $\mathbf{S}_{\sigma} \rightarrow \mathbf{S}_{\tau}$, the domain of continuous functions between $\mathbf{S}_{\sigma}$ and $\mathbf{S}_{\boldsymbol{\tau}}$. If $\mathbf{S}_{\tau}$ is the standard interpretation of the type $\tau$, then the standard interpretation of list $\tau$ is obtained by solving the recursive domain isomorphism:

$$
\text { list } \mathbf{S}_{\tau} \cong 1+\mathbf{S}_{\tau} \times\left(\text { list } \mathbf{S}_{\tau}\right)
$$

over the category of domains in the usual way [SP82]. The solution of this isomorphism can be represented informally by:

$$
\mathbf{S}_{(\text {list } \tau)}=\left\{\perp_{\mathbf{S}_{(\text {list } \tau)}}\right\} \bigcup\{\text { nil }\} \bigcup\left\{\text { cons } u l \mid u \in \mathbf{S}_{\tau}, l \in \mathbf{S}_{(\text {list } \tau)}\right\}
$$

with the ordering:

$$
\begin{aligned}
& \perp_{\mathbf{S}_{(l i s t r)}} \sqsubseteq l, \forall l \in \mathbf{S}_{(l i s t \tau)} \\
& (\text { cons } u l) \sqsubseteq\left(\text { cons } u^{\prime} l^{\prime}\right) \text { if } u \sqsubseteq u^{\prime} \text { and } l \sqsubseteq l^{\prime} \\
& n i l \sqsubseteq n i l
\end{aligned}
$$

The standard interpretation of $\Lambda_{T}$ is as usual, and is defined in Figure 6, with the standard interpretation of the constants in $\Lambda_{T}$ being given in Figure 7 .

\section{A Result Relating the Operational Semantics and Standard Interpretation of $\Lambda_{T}$}

Not all types are suitable as the type of a program. It is normal functional programming practice to restrict programs to being terms of ground type, that is, containing no function 


$$
\begin{aligned}
& \mathbf{S}_{(\sigma \rightarrow \tau)}=\left(\mathbf{S}_{\sigma}\right) \rightarrow\left(\mathbf{S}_{\tau}\right) \\
& \mathbf{S}_{(i s t \sigma)}=\text { list }\left(\mathbf{S}_{\sigma}\right)
\end{aligned}
$$

Interpretation of the Types

$\mathbf{S} \llbracket x^{\sigma} \rrbracket \rho^{\mathbf{S}}=\rho^{\mathbf{S}} x^{\sigma}$

$\mathbf{S} \llbracket c_{\sigma} \rrbracket \rho^{\mathbf{S}}=\mathbf{K}^{\mathbf{S}} \llbracket c_{\sigma} \rrbracket$

$\mathbf{S} \llbracket e_{1} e_{2} \rrbracket \rho^{\mathbf{S}}=\left(\mathbf{S} \llbracket e_{1} \rrbracket \rho^{\mathbf{S}}\right)\left(\mathbf{S} \llbracket e_{2} \rrbracket \rho^{\mathbf{S}}\right)$

$\mathbf{S} \llbracket \lambda x^{\sigma} . e \rrbracket \rho^{\mathbf{S}}=\lambda y_{\epsilon} \mathbf{S}_{\sigma} .\left(\mathbf{S} \llbracket e \rrbracket \rho^{\mathbf{S}}\left[y / x^{\sigma}\right]\right)$

$\mathbf{S} \llbracket \mathbf{f i x}_{(\sigma \rightarrow \sigma) \rightarrow \sigma} e \rrbracket \rho^{\mathbf{S}}=\bigsqcup_{i \geq 0}\left(\mathbf{S} \llbracket e \rrbracket \rho^{\mathbf{S}}\right)^{i} \perp_{\mathbf{S}_{\sigma}}$

Interpretation of the Language Constructs

Figure 6: The Standard Interpretation of $\Lambda_{T}$

$$
\begin{aligned}
& \mathrm{K}^{\mathrm{S}} \llbracket \text { true }_{\text {bool }} \rrbracket=\text { true } \quad \mathrm{K}^{\mathbf{S}} \llbracket \text { false }_{\text {bool }} \rrbracket=\text { false } \\
& \mathbf{K}^{\mathbf{S}} \llbracket \mathbf{i f}_{b o o l \rightarrow \sigma \rightarrow \sigma \rightarrow \sigma} \rrbracket x y z= \begin{cases}\perp_{\mathbf{S}_{\sigma}} & \text { if } x=\perp_{\mathbf{S}_{\text {bool }}} \\
y & \text { if } x=\text { true } \\
z & \text { if } x=\text { false }\end{cases} \\
& \mathbf{K}^{\mathbf{S}} \llbracket 0_{i n t} \rrbracket=0 \quad \mathbf{K}^{\mathbf{S}} \llbracket 1_{\text {int }} \rrbracket=1 \quad \ldots \\
& \mathrm{K}^{\mathrm{S}} \mathbb{\|}+_{\text {int } \rightarrow \text { int } \rightarrow \text { int }} \rrbracket=\lambda x_{\epsilon} \mathbf{S}_{\text {int }}, \lambda y \epsilon \mathrm{S}_{\text {int }}+x y \\
& \mathrm{~K}^{\mathrm{S}} \llbracket \operatorname{nil}_{(\text {list } \tau)} \rrbracket=n i l \\
& \mathbf{K}^{\mathbf{S}} \llbracket \operatorname{cons}_{\tau \rightarrow(\text { list } \tau) \rightarrow(\text { list } r)} \rrbracket=\text { cons }
\end{aligned}
$$

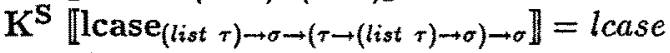

$$
\begin{aligned}
& \text { where } \\
& \text { lcase }: \mathbf{S}_{(\text {list } r)} \rightarrow \mathbf{S}_{\sigma} \rightarrow \mathbf{S}_{\tau \rightarrow(\text { list } \tau) \rightarrow \sigma} \rightarrow \mathbf{S}_{\sigma} \\
& \text { lcase } \perp_{\mathbf{S}_{(\text {ist } \mathrm{r})}} \text { sf } f=\perp_{\mathbf{S}_{\sigma}} \\
& \text { lcase nil s } f=s \\
& \text { lcase (cons } h t) s f=f h t
\end{aligned}
$$

Figure 7: The Standard Interpretation of the Constants in $\Lambda_{T}$ 
types. Of course subexpressions in the program can have function types, as long as the final type of a program does not. Formally, we define the predicates ground on types by:

$$
\begin{array}{llll}
\text { ground bool } & =\text { true } & \text { ground int } & =\text { true } \\
\text { ground }(\text { list } \sigma) & =\text { ground } \sigma & \text { ground }(\sigma \rightarrow \tau) & =\text { false }
\end{array}
$$

We now define a program to be any closed term of $\Lambda_{T}$ which has a ground type.

\section{Theorem 6.1 (Computational Adequacy Theorem)}

Given any program $e: \sigma, F_{\ell}$ the one-step reduction strategy which always chooses the leftmost redex, fails to terminate if and only if $\mathbf{S} \llbracket e \rrbracket\{\}=\perp_{\mathbf{S}_{\sigma}}$. Moreover, if the reduction sequence terminates in some $H N F e^{\prime}$, then

$$
\mathbf{S} \llbracket e \rrbracket\{\}=\mathbf{S} \llbracket e^{\prime} \rrbracket\{\} .
$$

where \{\} is the empty environment.

This theorem is proved by adapting the proof of a similar theorem for PCF, proved by Plotkin in [Plo77], and the proof can be found in [Bur91b, pp. 161ff]. Part of the proof also shows that $\beta \delta$-reduction preserves the standard interpretation of programs.

\section{Evaluators and the Correctness of the Evaluation Transformer Model of Reduction}

Most works on the semantics of programming languages have only one concept of an infinite computation, namely that which never terminates, and produces no result. In denotational semantics, this is usually given the value $\perp$ (from the appropriate domain). In order to deal with evaluators, we need a more general concept of a non-terminating computation. Consider the evaluator $\xi_{T}$ informally introduced in Section 2. The intuition about this evaluator is that it needs to evaluate the structure of its argument list, that is, if it evaluates an expression to the form (cons $u l$ ), then it needs to evaluate $l$ with $\xi_{T}$. One way of implementing this evaluator is to evaluate the whole of the structure of the list before returning any result. If the list is infinite, or has an undefined tail at some point, then this implementation of the evaluator will fail in the classical way - it will produce no output. Another way to implement the evaluator is to evaluate the expression to the form (cons $u l$ ), return this much of the result, and then begin evaluating $l$ with $\xi_{T}$. Thus, at any finite time, the infinite computation can have produced a finite approximation to the expression being evaluated. We therefore make the following definition.

\section{Definition 7.1}

An infinite computation is an infinite reduction sequence. When the reduction of an expression fails to terminate in the classical sense, producing no HNF, then we will say that it produces no output. 
We will be very careful in our use of these phrases, so that an infinite computation refers only to the property that it is an infinite reduction sequence, and does not determine the amount of the result that has been produced.

Because $\beta \delta$-reduction preserves the standard interpretation of programs, in changing the reduction strategy for a program we need only concern ourselves with preserving the termination properties of the new reduction strategy for the program. For the language $\Lambda_{T}$, the leftmost reduction strategy has the property that it produces no output for a program if and only if the program has no HNF (Computational Adequacy Theorem). Any change in the reduction model should preserve this property. With this in mind, we make the following definition.

\section{Definition 7.2}

A reduction strategy is safe for a program if it initiates an infinite computation if and only if the program has no HNF. Furthermore, if the program has no HNF, then no output should be produced from the program.

We will say it is safe to evaluate some term (with a particular evaluator) if the resulting reduction strategy is safe.

We say 'initiates' in the above definition because the definition must cover both sequential and parallel implementations. In a sequential implementation, there will be only one reduction sequence, and so 'initiates an infinite reduction sequence' is equivalent to 'consists of an infinite reduction sequence'. A parallel reduction strategy allows one reduction sequence to initiate the parallel reduction of some other expression. On a parallel machine, it is still possible that the HNF of a program could be reached even if some processor was performing an infinite reduction sequence. This is ruled out by the above definition of safety. Sometimes what we have called a safe reduction strategy is called conservative, to distinguish them from speculative ones, which might do some evaluation of expressions which do not need to be evaluated in order to produce the HNF of a program. Peyton Jones discusses some of the issues involved in choosing between a conservative and speculative evaluation model [Pey89]. It is much simpler to implement a conservative parallel evaluation strategy than a speculative one because there are no useless processes which have to be garbage collected. A complete specification of an abstract machine employing speculative evaluation can be found in [Par91].

An evaluator captures the intuitive notion of doing a certain amount of evaluation to an expression. The important property of an evaluator is the way it divides the expressions of a type into two classes: those for which it terminates, and those for which it initiates an infinite computation. If an evaluator fails to terminate when reducing an expression $e$, then it is natural that it should fail to terminate when evaluating any expression whose standard interpretation is less defined than that of $e$. The concept of 'all the values at most as defined as' some other value is conveniently captured by the notion of a Scott-closed set. A set $S \subseteq \mathrm{S}_{\sigma}$ is $S$ cott-closed if:

1. $x \sqsubseteq s \in S$ then $x \in S$, and

2. $X \subseteq S$ and $X$ is directed, then $\sqcup X \in S$. 
We can now formally define what we mean by an evaluator.

\section{Definition 7.3}

An evaluator is a reduction strategy. An evaluator corresponding to a Scott-closed set $^{4} S \subseteq \mathbf{S}_{\sigma}$ fails to terminate when evaluating an expression $e: \sigma$ if and only if $\left(\mathbf{S} \llbracket e \rrbracket \rho^{S}\right) \in S$. We will write this as $\xi_{S}^{\sigma}$. For notational convenience, $\xi_{\{\}}^{\sigma}$, which does no evaluation, will be written as $\xi_{N O}$, dropping the type superscript.

Recalling that reduction preserves the standard interpretation of expressions (from the proof of the Computational Adequacy Theorem), if the evaluator terminates, then the standard interpretation of the expression will be unchanged. Even though there may be several reduction strategies which fail to terminate when evaluating an expression if and only if its standard interpretation is in the Scott-closed set $S$, they all have the essential property that they fail to terminate, or terminate with a result whose standard interpretation is the same as that of the original expression. We therefore feel justified in writing the evaluator $\xi_{S}^{\sigma}$.

Even though the definition of an evaluator is quite abstract, it is sometimes easy to give reduction strategies which implement particular evaluators. For example, $\left\{1_{S_{\sigma}}\right\}$ is a Scott-closed set, and the Computational Adequacy Theorem states that leftmost reduction fails to terminate (producing no result) for an expression if and only if the standard interpretation the expression is $\perp_{\mathbf{S}_{\sigma}}$. Thus leftmost reduction is an example of a reduction strategy which satisfies the conditions for $\xi_{\perp_{\mathrm{S}_{\sigma}}^{\sigma}}$ for any type $\sigma$. In Section 8.3 we will see how the evaluators discussed informally in Section 2 arise naturally from a particular abstract interpretation.

We would like to annotate all function applications in a program with evaluation transformers. The following theorem shows how this can be done.

\section{Theorem 7.4 (Context-Sensitive Evaluation Transformer Theorem)}

Suppose that $f: \sigma_{1} \rightarrow \ldots \rightarrow \sigma_{n} \rightarrow \tau$, and that $S_{i}$, for some $i$, and $T$ are $S$ cott-closed subsets of $\mathbf{S}_{\sigma_{i}}$ and $\mathbf{S}_{\tau}$ respectively. If in an application

$$
f e_{1} \ldots e_{n}
$$

we have that $\left(\mathbf{S} \llbracket e_{i} \rrbracket \rho^{\mathbf{S}}\right) \in S_{i}$ implies that $\left(\mathbf{S} \llbracket f e_{1} \ldots e_{n} \rrbracket \rho^{\mathbf{S}}\right) \in T$, then when it is safe to evaluate $\left(f e_{1} \ldots e_{n}\right)$ with $\xi_{T}^{\tau}$, then it is safe to evaluate $e_{i}$ with $\xi_{S_{i}}^{\sigma_{i}}$.

\section{Proof}

There are two cases to consider:

\footnotetext{
${ }^{4}$ We use a Scott-closed set rather than simply a left-closed set because we want evaluators to have a sort of continuity property. For example, using a Scott-closed set ensures that the evaluator which fails to terminate in evaluating all expressions whose standard interpretations are lists with undefined tails will also fail to terminate when evaluating those which denote infinite lists, enforced by part (2) of the definition of Scott-closed sets. Left-closure is not sufficient.
} 
1. Evaluation of $e_{i}$ with $\xi_{S_{i}}^{\sigma_{i}}$ terminates: This is trivially safe.

2. Evaluation of $e_{i}$ with $\xi_{S_{i}}^{\sigma_{i}}$ initiates an infinite computation: From the definition of $\xi_{S_{i}}^{\sigma_{i}}$, this can only happen if $\left(\mathbf{S} \llbracket e_{i} \rrbracket \rho^{\mathbf{S}}\right) \in S_{i}$. Therefore, by a condition of the theorem, $\left(\mathbf{S} \llbracket f e_{1} \ldots e_{n} \rrbracket \rho^{\mathbf{S}}\right) \in T$, and so evaluation of the application with $\xi_{T}^{\tau}$ will initiate an infinite computation. Since it was safe to evaluate the application with $\xi_{T}^{\tau}$, this means that the program has no HNF, and so it was safe to initiate an infinite computation when evaluating the expression $e_{i}$ with $\xi_{S_{i}}^{\sigma_{i}}$

The default reduction strategy is $F_{\ell}$. By the Computational Adequacy Theorem, this is a safe reduction strategy for a program. We will see in Section 8 how the behaviour of functions over Scott-closed sets can be determined using abstract interpretation, giving a technique for finding evaluation transformers that can be implemented in a compiler.

Unfortunately, this theorem does not give all the information we might want in an implementation. If $x_{j}$ is some formal parameter to a function, the expression $\left(x_{j} e_{1} \ldots e_{n}\right.$ : $\tau)$ cannot be annotated with evaluation transformers because in general we do not know which function $x_{i}$ will be bound to at compile-time. However, at run-time, $x_{i}$ will be bound to an expression, which, if reduced, will eventually reduce to a partial application of a function (or fail to terminate). At this stage, we could use some of the properties of the function to allow some evaluation of the expression $e_{i}$. Because at compile-time we do not know the complete context of the function being applied, the evaluation transformer information that is used must be true in any context. This information is given by the following theorem.

\section{Theorem 7.5 (Context-Free Evaluation Transformer Theorem)}

Suppose that $f: \sigma_{1} \rightarrow \ldots \sigma_{n} \rightarrow \tau$ and that $S_{i}$, for some $i$, and $T$ are Scott-closed sets of $\mathbf{S}_{\sigma_{i}} \mathbf{S}_{\tau}$ respectively, and that $\forall j \in\{1, \ldots, n\}, j \neq i, \forall e_{j}: \sigma_{j}$, whenever $\left(\mathbf{S} \llbracket e_{i} \rrbracket \rho^{\mathbf{S}}\right) \in S_{i}$, then

$$
\mathbf{S} \llbracket f e_{1} \ldots e_{n} \rrbracket \rho^{\mathbf{S}} \in T .
$$

Then for any application of $f$, when it is safe to evaluate the application with $\xi_{T}^{\tau}$, then it is safe to evaluate the ith argument with $\xi_{S_{i}}^{\sigma_{i}}$.

\section{Proof}

This is proved in the same way as Theorem 7.4.

We call the evaluation transformers determined using Theorem 7.5 context-free evaluation transformers because they are safe in any context. The concept of context-free information was first introduced in [HBP86, HBP88], and context-free evaluation transformers in [Bur87b]. In a similar way, we say that the evaluation transformers determined using Theorem 7.4 are context-sensitive since they depend on the context of the other 
arguments in the function application. It should be intuitively clear that taking the context of an argument expression into account may allow more evaluation than just using the context-free evaluation transformer information, so that context-sensitive evaluation transformers are needed. If not, using the abstract interpretation in the next section, calculate the context-sensitive evaluation transformer for the expression $e$ in $\operatorname{map}\left(\lambda x^{\sigma} . x\right) e$ and compare it with the context-free evaluation transformer for the second argument of map. This example is discussed further in [Bur87b, Section 4.2] and [Bur91b, Section 5.3], and the uses of the two different types of evaluation transformer information in compiling lazy functional programs is discussed in [Bur91a, Section 6] and [Bur91b, Chapter 6].

\section{Abstract Interpretation and Evaluation Transform- ers}

The work of the previous section would be little more than a theoretical curiousity if we had no way of determining the behaviour of functions on arguments from particular Scottclosed sets. Fortunately, abstract interpretations can be developed which characterise the behaviour of functions on particular Scott-closed sets. They are called abstract interpretations because they abstract away from many of the details of the standard interpretation in order to focus on finding out information about a particular property, and an object having a particular property is represented by its belonging to a particular Scott-closed set.

Our plan then is to give a parameterised abstract interpretation. Given parameters which satisfy certain conditions, a theorem shows how calculations in the abstract domain give results about the Scott-closed set behaviour of the standard interpretation of a function. This information can then be used to determine evaluation transformers which result in safe reduction strategies, using Theorems 7.4 and 7.5. To conclude the section, we give an example of an abstract interpretation which can be used to determine the evaluation transformers intuitively motivated in Section 2. The work of this section is not new, see [Bur87a, Bur87b] for example, although the decoupling of evaluators from abstract interpretation, and the slant of this presentation is.

\subsection{A Parameterised Abstract Interpretation of $\Lambda_{T}$}

A parameterised abstract interpretation of $\Lambda_{T}$ is given in Figure 8. To define a particular abstract interpretation, the following have to be provided:

- finite lattices as the abstract interpretation of the types bool, int and (list $\tau$ ) for any type $\tau$,

- a strict, continuous abstraction map for each of the types bool, int and (list $\tau$ ), from the standard interpretation of the type to its abstract interpretation, and

- an abstract interpretation for each of the constants in $\Lambda_{T}$ which satisfies the constant safety condition. 


$$
\begin{aligned}
& \left.\begin{array}{l}
\mathbf{A}_{\text {bool }} \\
\mathbf{A}_{\text {int }} \\
\mathbf{A}_{(i \text { ist } \sigma)}=\operatorname{LIST} \mathbf{A}_{\sigma}
\end{array}\right\} \begin{array}{l}
\text { For each specific abstract interpretation, } \\
\text { finite lattices must be given for these. }
\end{array} \\
& \mathbf{A}_{(\sigma \rightarrow \tau)}=\left(\mathbf{A}_{\sigma}\right) \rightarrow\left(\mathbf{A}_{\tau}\right)
\end{aligned}
$$

Interpretation of the Types

$\mathbf{A} \llbracket x^{\sigma} \rrbracket \rho^{\mathbf{A}}=\rho^{\mathbf{A}} x^{\sigma}$

$\mathbf{A} \llbracket c_{\sigma} \rrbracket \rho^{\mathbf{A}}=\mathbf{K}^{\mathbf{A}} \llbracket c_{\sigma} \rrbracket-$ Must be given for each specific abstract interpretation.

$\mathbf{A} \llbracket e_{1} e_{2} \rrbracket \rho^{\mathbf{A}}=\left(\mathbf{A} \llbracket e_{1} \rrbracket \rho^{\mathbf{A}}\right)\left(\mathbf{A} \llbracket e_{2} \rrbracket \rho^{\mathbf{A}}\right)$

$\mathbf{A} \llbracket \lambda x^{\sigma} . e \rrbracket \rho^{\mathbf{A}}=\lambda y_{c} \mathbf{A}_{\sigma} \cdot\left(\mathbf{A} \llbracket e \rrbracket \rho^{\mathbf{A}}\left[y / x^{\sigma}\right]\right)$

$\mathbf{A} \llbracket \operatorname{fix}_{(\sigma \rightarrow \sigma) \rightarrow \sigma} e \rrbracket \rho^{\mathbf{A}}=\bigsqcup_{i \geq 0}\left(\mathbf{A} \llbracket e \rrbracket \rho^{\mathbf{A}}\right)^{i} \perp_{\mathbf{A}_{\sigma}}$

Interpretation of the Language Constructs

Figure 8: The Abstract Interpretation of $\Lambda_{T}$

The abstraction maps are used to relate the standard and abstract interpretations of $\Lambda_{T}$, and should be defined in such a way as to capture the property of interest. Given abstraction maps for the listed types, abstraction maps can be defined inductively for the higher types [Bur87b, pp. 36ff.], [Bur91b, pp. 68ff.].

Abstract domain points can be regarded as representing a Scott-closed set of objects from the standard interpretation of a type. For any type $\sigma$, the representation function, $\gamma_{\sigma}$, called concretisation, is defined by:

$$
\gamma_{\sigma} \bar{s}=\left\{s \mid\left(\alpha_{\sigma} s\right) \sqsubseteq \bar{s}\right\}
$$

where $\alpha_{\sigma}$ is the abstraction map for the type $\sigma$. It can be proved that $\gamma_{\sigma}$ is well-defined and continuous, and that $\left(\gamma_{\sigma} \bar{s}\right)$ is a Scott-closed subset of the standard interpretation of $\sigma$ for any $\bar{s}$ in an abstract interpretation of the type $\sigma$ [Bur87b, Lemma 2.2.8],[Bur91b, Lemma 3.2.4].

Abstract domains must be lattices because the abstraction map for functional types involves the taking of least upper bounds; finiteness ensures the computability of the abstract interpretation.

Note that we have written the abstract interpretation of the type (list $\sigma$ ) as LIST $\mathbf{A}_{\sigma}$. This is because the abstract domain for (list $\sigma$ ) is allowed to depend on the abstract domain for the type $\sigma$. 
The abstract interpretation of a constant $c_{\sigma}, \mathrm{K}^{\mathbf{A}} \llbracket c_{\sigma} \rrbracket$, satisfies the constant safety condition if

$$
\mathbf{K}^{\mathbf{A}} \llbracket c_{\sigma} \rrbracket \sqsupseteq \alpha_{\sigma}\left(\mathbf{K}^{\mathbf{S}} \llbracket c_{\sigma} \rrbracket\right) .
$$

\subsection{A Relationship Between the Standard and Abstract Inter- pretations of $\Lambda_{T}$}

The following theorem relates results obtained using an abstract interpretation of $\Lambda_{T}$ to its standard interpretation. It can be used in determining evaluation transformers.

\section{Theorem 8.1 (Correctness Theorem for Abstract Interpretation)}

Suppose that we have:

- finite lattices as the abstract interpretation of the types bool, int and (list $\tau$ ) for any type $\tau$,

- a strict, continuous abstraction map for each of the types bool, int and (list $\tau)$, from the standard interpretation of the type to its abstract interpretation,

- abstract interpretations of constants which satisfy the constant safety condition, and

- environments $\rho^{\mathbf{A}}$ and $\rho^{\mathbf{S}}$ such that for all $x^{\tau},\left(\rho^{\mathbf{A}} x^{\tau}\right) \sqsupseteq \alpha_{\tau}\left(\rho^{\mathbf{S}} x^{\tau}\right)$.

Then for any $f: \sigma \rightarrow \tau$, we have that if $\overline{\mathcal{s}} \in \mathbf{A}_{\sigma}$ and $\left(\mathbf{A} \llbracket f \rrbracket \rho^{\mathbf{A}}\right) \bar{s} \sqsubseteq \bar{t}$, then for all $s \in\left(\gamma_{\sigma} \bar{s}\right),\left(\left(\mathbf{S} \llbracket f \rrbracket \rho^{\mathbf{S}}\right) s\right) \in\left(\gamma_{\tau} \bar{t}\right)$.

When a function $f$ has the type $\sigma_{1} \rightarrow \ldots \rightarrow \sigma_{n} \rightarrow \tau$, then the following theorem gives a useful result about an application of the function, and is useful for determining evaluation transformers.

\section{Theorem 8.2}

Suppose that $f: \sigma_{1} \rightarrow \ldots \rightarrow \sigma_{n} \rightarrow \tau$ and $\left.(\mathbf{A} \llbracket f] \rho^{\mathbf{A}}\right) \bar{s}_{1} \ldots \bar{s}_{n} \subseteq \bar{t}$. Then $\forall j \in\{1, \ldots, n\}, j \neq i, \forall e_{j}: \sigma_{j}$ such that $\left(\mathbf{A} \llbracket e_{j} \rrbracket \rho^{\mathbf{A}}\right) \sqsubseteq \bar{s}_{j}, \forall s_{i} \in\left(\gamma_{\sigma_{1}} \bar{s}_{i}\right)$,

$\left(\mathbf{S} \llbracket f \rrbracket \rho^{\mathbf{S}}\right)\left(\mathbf{S} \llbracket e_{1} \rrbracket \rho^{\mathbf{S}}\right) \ldots\left(\mathbf{S} \llbracket e_{i-1} \rrbracket \rho^{\mathbf{S}}\right) s_{i}\left(\mathbf{S} \llbracket e_{i+1} \rrbracket \rho^{\mathbf{S}}\right) \ldots\left(\mathbf{S} \llbracket e_{n} \rrbracket \rho^{\mathbf{S}}\right) \in\left(\gamma_{\tau} \bar{t}\right)$.

\subsection{An Example Abstract Interpretation}

The purpose of this section is to define a particular abstract interpretation which can be used to determine the evaluation transformers motivated in Section 2. Our intuitions about the standard interpretation of the expressions for which these evaluators fail to terminate can be used to define abstract domain points.

For any type which has a flat domain as its standard interpretation, such as bool and int, HNF is the same as normal form, and so the only amount of evaluation that can be done to expressions is to HNF. The Computational Adequacy Theorem states that evaluation of an expression to HNF will fail to terminate if and only if the standard 
interpretation of the expression is bottom. Therefore we need an abstract domain point representing the Scott-closed set $\{\perp\}$. We also need an abstract domain point representing the whole standard interpretation of the type. (Recall the 'representing' is formalised using concretisation.)

\section{Definition 8.3}

If $B$ is bool or int, then $\mathbf{A}_{B}=\mathbf{2}$, where $\mathbf{2}=\{0,1\}$ with the ordering defined by $0 \sqsubseteq 1$.

The abstraction map is defined by:

$$
\begin{aligned}
& \alpha_{B}: \mathbf{S}_{B} \rightarrow \mathbf{2} \\
& \alpha_{B} u= \begin{cases}0 & \text { if } u=\perp_{\mathbf{S}_{B}} \\
1 & \text { otherwise }\end{cases}
\end{aligned}
$$

This abstract domain was first introduced by Alan Mycroft [Myc80, Myc81].

For lists, our intuition about the evaluator $\xi_{T}$ is that it fails to terminate if and only if the standard interpretation of the expression being reduced is an infinite list, or a list ending in bottom (including the bottom list), and $\xi_{T H}$ fails to terminate in the further case that the expression has a finite list with at least one bottom element in it as its standard interpretation. We therefore make the following definition.

\section{Definition 8.4}

For all types $\tau$, the domain $\mathbf{A}_{(\text {list } \tau)}$ contains the four elements $\{\perp, \infty, \perp \in, T \in\}$, where the ordering is defined by $\perp \sqsubseteq \infty \sqsubseteq \perp \in \sqsubseteq T \in$.

The abstraction map is defined by:

$$
\begin{aligned}
& \alpha_{(\text {list } \tau)}: \mathbf{S}_{(\text {list } \tau)_{1}} \rightarrow \mathbf{A}_{(\text {list } \tau)} \\
& \alpha_{(\text {list } \tau)} u=\left\{\begin{array}{cll}
\perp & \text { if } & u=\perp_{\mathbf{S}_{(\text {list } \tau)}} \\
\infty & \text { if } & T u=\perp_{\mathbf{S}_{(\text {list } \tau)}} \wedge u \neq \perp_{\mathbf{S}_{(\text {list } \tau)}} \\
\perp \in & \text { if } & F u=\perp_{\mathbf{S}_{(\text {ist } \tau)}} \wedge T u \neq \perp_{\mathbf{S}_{(\text {list } \tau)}} \\
T \in & \text { if } & F u \neq \perp_{\mathbf{S}_{(\text {list } \tau)}}
\end{array}\right. \\
& \text { where }
\end{aligned}
$$

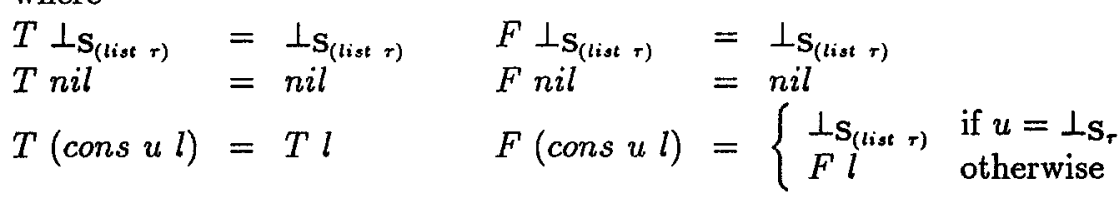

This abstract domain is due to Phil Wadler [Wad87].

Abstract interpretations of the constants in $\Lambda_{T}$ using these abstract domains, and satisfying the constant safety condition, can be found in Figure 9. The abstract interpretation of functions defined in $\Lambda_{T}$ can then be determined using the abstract interpretation defined in Figure 8. Some examples are given in Figure 10.

In using Theorem 7.5 in determining context-free evaluation transformers, the point $T_{\mathbf{A}_{\sigma_{j}}}$ can be used in the test of Theorem 8.2 for all $j \neq i$ because $\forall e_{j}: \sigma_{j},\left(\mathbf{A} \llbracket e_{j} \rrbracket \rho^{\mathbf{A}}\right) \sqsubseteq$ 
$\mathbf{K}^{\mathrm{A}} \llbracket$ true $_{b o o l} \rrbracket=1=\mathbf{K}^{\mathrm{A}} \llbracket$ false $_{b o o l} \rrbracket$

$\mathbf{K}^{\mathbf{A}} \llbracket \mathbf{i f}_{\text {bool } \rightarrow \sigma \rightarrow \sigma \rightarrow \sigma} \rrbracket x y z= \begin{cases}\perp_{\mathbf{A}_{\sigma}} & \text { if } x=\perp_{\mathbf{A}_{\text {boot }}}(=0) \\ y \sqcup z & \text { otherwise }\end{cases}$

$\mathrm{K}^{\mathrm{A}} \llbracket \mathbf{0}_{\text {int }} \rrbracket=1=\mathrm{K}^{\mathrm{A}} \llbracket \mathbf{1}_{\text {int }} \rrbracket=\ldots$

$\mathrm{K}^{\mathrm{A}} \llbracket+_{\text {int } \rightarrow \text { int } \rightarrow \text { int }} \rrbracket x y=x \sqcap y$

$\mathbf{K}^{\mathbf{A}} \llbracket$ nil $_{(\text {ist } r)} \rrbracket=T \in$

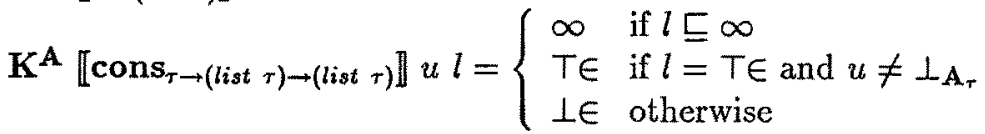

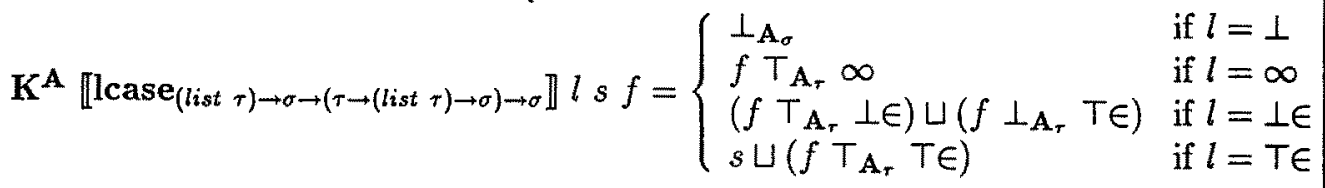

Figure 9: The Abstract Interpretation of Constants in $\Lambda_{T}$

\begin{tabular}{|c|c|c|}
\hline $\bar{u}$ & $\overline{\text { length }} \bar{u}$ & $\overline{\text { sumlist }} \bar{u}$ \\
\hline$\perp$ & 0 & 0 \\
$\infty$ & 0 & 0 \\
$\perp \in$ & 1 & 0 \\
$T \epsilon$ & 1 & 1 \\
\hline
\end{tabular}

For all types $\tau$, append $:($ list $\tau) \rightarrow($ list $\tau) \rightarrow($ list $\tau)$

$\overline{\text { append } \overline{x s} \overline{y s}:}$\begin{tabular}{|c|c|c|c|c|c|}
\hline$\overline{y s}$ & $\perp$ & $\infty$ & $\perp \in$ & $T \in$ \\
\hline$\overline{x s}$ & & & & \\
\hline$\perp$ & $\perp$ & $\perp$ & $\perp$ & $\perp$ \\
$\infty$ & $\infty$ & $\infty$ & $\infty$ & $\infty$ \\
$\perp \epsilon$ & $\infty$ & $\infty$ & $\perp \epsilon$ & $\perp \epsilon$ \\
\hline$\epsilon$ & $\infty$ & $\infty$ & $\perp \epsilon$ & $T \epsilon$ \\
\hline
\end{tabular}

Figure 10: The Abstract Interpretation of Some Example Functions 


\begin{tabular}{|c|c|c|}
\hline$E$ & $L E N G T H_{1} E$ & $S U M L I S T_{1} E$ \\
\hline $\begin{array}{c}\xi_{N O} \\
\left.\xi_{\left(\gamma_{\text {int }}\right.}^{\text {int }}\right)\end{array}$ & 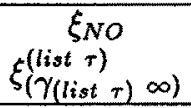 & $\begin{array}{c}\xi_{N O} \\
\xi_{\left(\gamma_{(l i s t}^{(\text {list } \tau)}\right)}^{(\text {list })}\end{array}$ \\
\hline
\end{tabular}

\begin{tabular}{|c|c|c|c|c|}
\hline$E$ & $C_{C O N S_{1} E}$ & $\mathrm{CONS}_{2} E$ & $A P P E N D_{1} E$ & $A P P E N D_{2} E$ \\
\hline$\xi_{N O}$ & $\xi_{N O}$ & $\xi_{N O}$ & $\xi_{N O}$ & $\xi_{N O}$ \\
\hline$\xi_{(\gamma(\text { list }}^{(\text {list } r)}$ & $\xi_{N O}$ & $\xi_{N O}$ & $\xi_{(\gamma(\text { list } \tau)}^{(\text {list })}$ & $\xi_{N O}$ \\
\hline$\xi^{(\text {list } \tau)}$ & & $\xi^{(\text {list } \tau)}$ & $\varepsilon_{(\text {list } \tau)}^{(\text {lis }}$ & $\xi^{(\text {list } \tau)}$ \\
\hline$\left.\zeta\left(\gamma_{(\text {list } \tau)}\right) \infty\right)$ & \$NO & $\left.s\left(\gamma_{(\text {list }} \tau\right) \infty\right)$ & $s(\gamma($ list $\tau) \infty)$ & $S(\gamma($ list $\tau) \infty)$ \\
\hline$\xi_{\left(\gamma_{(\text {list } \tau) ~}^{(\text {list } \tau)}\right)}^{(k)}$ & $\xi_{\left(\gamma_{\tau} \perp_{\mathbf{A}_{\tau}}\right)}$ & $\xi_{(\gamma(\text { list } \tau)}^{(\text {list } \tau)}$ & $\xi_{\left(\gamma_{(\text {list } \tau)}^{(\text {list } \tau)}\right.}$ & $\xi_{(\gamma(\text { list } \tau)}^{(\text {list } \tau)}$ \\
\hline
\end{tabular}

Figure 11: Context-Free Evaluation Transformers for Some Example Functions Using this Abstract Interpretation

$T_{\mathbf{A}_{\sigma_{j}}}$. The context-free evaluation transformers for some functions are recorded in Figure 11 .

We must emphasise that this section is by way of an example; choosing different abstract domains for these types means that different evaluators are defined, and hence different evaluation transformers can be determined. Some further examples of abstract domains for these types, including ones which take into account the types of the elements in lists, can be found in [Bur91b, Chapter 4].

\subsection{Obtaining Operational Definitions of Evaluators}

The definition of evaluators given in Definition 7.3 is rather abstract, but now we can give more concrete operational definitions of them. From Figure 11 we can see that in an application of $\operatorname{cons}_{\tau \rightarrow(\text { list } \tau) \rightarrow(\text { list } \tau)}$ being evaluated with $\xi_{\left(\gamma_{(\text {list } \tau)}\left(\text { list }_{1}\right)\right.}$, the second argument to cons $s_{\tau \rightarrow(\text { list } \tau) \rightarrow(\text { list } \tau)}$, its tail, needs to be evaluated with $\xi_{T}$, corresponding

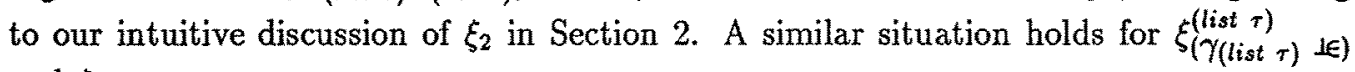
and $\xi_{T H}$.

This gives rise to a general methodology for determining evaluators for a particular type: Given the desired evaluators, determine the evaluation transformers for the constructors of the type, which then show how to implement the evaluators of that type.

\section{Conclusion and Further Work}

In this paper we have given an evaluation model for lazy functional languages which is more efficient than lazy evaluation, and can be used to run programs on sequential and parallel machines. We have shown how to obtain the information needed for the model 
from a semantically sound analysis technique, which can be implemented in a compiler [CP85, MH87, Hun89]. The resulting evaluation model has been proved correct.

The theory developed so far has not taken into account the fact that most implementations of lazy functional languages use some form of graph reduction [Wad71, Chapter 4],[Pey87]. In graph reduction, expressions are represented as graphs. When an application is reduced, repeated copies of a variable in the term being substituted for are replaced by pointers to a single copy of the graph for the argument expression. Using this, it can be ensured that an expression is evaluated at most once. Each reduction of such a shared expression is equivalent to doing several reductions at once of the expression of which it is a part. Graph reduction has been formalised in $[\mathrm{BvEG}+87]$, and it has been proved that this gives a safe reduction strategy. We need to adapt the results to a model which uses evaluation transformer information.

A second way in which we need to develop our theory is to use the results available from projection analysis [WH87]. A projection is an idempotent function which is at most defined as the identity function. In [Bur90], we showed how evaluation transformers could be determined from the results of projection analysis, but the development was not as rigorous as that contained in this paper. The key problem is that some the evaluators corresponding to some projections cannot be formalised as reduction strategies in the sense that we have used the term in this paper, and the canonical example of such a projection is $H$, defined by:

$$
\begin{aligned}
H \perp_{\mathbf{S}_{(\text {list r) }}} & =\perp_{\mathbf{S}_{(i s t r)}} \\
H \text { nil } & =\text { nil } \\
H(\text { cons } u l) & = \begin{cases}\perp_{\mathbf{S}_{(i s t ~ \tau)}} & \text { if } u=\perp_{\mathbf{S}_{\tau}} \\
\text { cons } u(H l) & \text { otherwise }\end{cases}
\end{aligned}
$$

In [Bur90] we defined an evaluator corresponding to a projection $\beta: \sigma \rightarrow \sigma$ to be a 'reduction strategy' such that, given any expression $e: \sigma$ :

1. it initiates an infinite computation when evaluating $e$ if and only if $\beta\left(\mathbf{S} \llbracket e \rrbracket \rho^{\mathbf{S}}\right)=$ $\perp_{\mathrm{S}_{\sigma}} ;$ and

2. if the evaluation of an expression $e$ terminates with expression $e^{\prime}$, then we have that

$$
\mathbf{S} \llbracket e^{\prime} \rrbracket \rho^{\mathbf{S}}=\beta\left(\mathbf{S} \llbracket e \rrbracket \rho^{\mathbf{S}}\right) .
$$

Unfortunately, the use of the term 'reduction strategy' is not consistent with how we have used it in this paper, and furthermore, we currently have not developed a way of formalising what is meant. The reason why it is not a reduction strategy is that the evaluation of an expression using one of these evaluators may change the value of an expression. To see this, note that by the definition of an evaluator corresponding to $H$, when the argument to $H$ is an application of cons, and the first argument of cons is not $\perp_{S_{r}}$, the value of the expression has to be changed, even though its reduction terminates.

\section{Acknowledgements}

Much of this paper was produced by directly lifting text out of the book [Bur91b], which has been done with the kind permission of the publisher. 
I have greatly appreciated the many discussions I have had with my colleagues at Imperial College, Sebastian Hunt, Thomas Jensen and David Sands in particular, whilst working on formalising evaluators and the evaluation transformer model. Sebastian suggested I should decouple the definition of evaluators from abstract domain points, associating them instead with Scott-closed sets. Thanks to Henk Barendregt for suggesting I contact Roger Hindley concerning proving that leftmost reduction is head-normalising, and to Roger for the discussions we had over the proof.

This work is based on the research I have been doing over a number of years. The many people who were associated are acknowledged in the papers referenced by this one.

\section{References}

[Abr90] S. Abramsky. The lazy lambda calculus. In D.A. Turner, editor, Research Topics in Functional Programming, chapter 4, pages 65-116. Addison-Wesley, 1990.

[Bar84] H.P. Barendregt. The Lambda Calculus, volume 103 of Studies in Logic and the Foundations of Mathematics. Elsevier Science Publishers B.V., P.O. Box 1991, 1000 BZ Amsterdam, The Netherlands, 2nd edition, 1984.

[Bur87a] G. L. Burn. Evaluation transformers - A model for the parallel evaluation of functional languages (extended abstract). In G. Kahn, editor, Proceedings of the Functional Programming Languages and Computer Architecture Conference, pages 446-470. Springer-Verlag LNCS 274, September 1987.

[Bur87b] G.L. Burn. Abstract Interpretation and the Parallel Evaluation of Functional Languages. $\mathrm{PhD}$ thesis, Imperial College, University of London, March 1987.

[Bur90] G.L. Burn. Using projection analysis in compiling lazy functional programs. In Proceedings of the 1990 ACM Conference on Lisp and Functional Programming, pages 227-241, Nice, France, 27-29 June 1990.

[Bur91a] G.L. Burn. Implementing the evaluation transformer model of reduction on parallel machines. Journal of Functional Programming, 1(2), April 1991. To appear. Also appears as Department of Computing Report Number 90/24, Imperial College, LONDON SW7 2BZ.

[Bur91b] G.L. Burn. Lazy Functional Languages: Abstract Interpretation and Compilation. Research Monographs in Parallel and Distributed Computing. Pitman in association with MIT Press, 1991. To appear.

$[$ BvEG+87] H.P. Barendregt, M.C.J.D. van Eekelen, J.R.W. Glauert, J.R. Kennaway, M.J. Plasmeijer, and M.R. Sleep. Term graph rewriting. In Proceedings of PARLE 87, volume 2, pages 141-158. Springer-Verlag LNCS 259, Eindhoven, The Netherlands, June 1987. 
[CP85] C. Clack and S.L. Peyton Jones. Strictness analysis - a practical approach. In J.-P. Jouannaud, editor, Proceedings of the Functional Programming Languages and Computer Architecture Conference, pages 35-49. Springer-Verlag LNCS 201, September 1985.

[FH88] A.J. Field and P.G. Harrison. Functional Programming. Addison-Wesley International Computer Science Series, 1988.

[HBP86] C.L. Hankin, G.L. Burn, and S.L. Peyton Jones. A safe approach to parallel combinator reduction (extended abstract). In Proceedings ESOP 86 (European Symposium on Programming), pages 99-110, Saabrucken, Federal Republic of Germany, March 1986. Springer-Verlag LNCS 213.

[HBP88] C.L. Hankin, G.L. Burn, and S.L. Peyton Jones. A safe approach to parallel combinator reduction. Theoretical Computer Science, 56:17-36, 1988.

[Hin78] R. Hindley. Standard and normal reductions. Transactions of the American Mathematical Society, 241:253-271, July 1978.

[Hun89] S. Hunt. Frontiers and open sets in abstract interpretation. In Proceedings of the Conference on Functional Programming Languages and Computer Architecture, Imperial College, London, 11-13 September 1989.

[MH87] C. Martin and C. Hankin. Finding fixed points in finite lattices. In G. Kahn, editor, Proceedings of the Functional Programming Languages and Computer Architecture Conference, pages 426-445. Springer-Verlag LNCS 274, September 1987.

[Mit76] G. Mitschke. $\lambda$-kalkül, $\delta$-konversion und axiomatische rekursionstheorie. Technical Report 274, Fachbereit Mathematik, Technische Hochshule, Darmstadt, 1976. 77 pages.

[Myc80] A. Mycroft. Theory and practice of transforming call-by-need into call-byvalue. In 4th International Symposium on Programming, pages 269-281, Paris, April 1980. Springer-Verlag LNCS 83.

[Myc81] A. Mycroft. Abstract Interpretation and Optimising Transformations for Applicative Programs. $\mathrm{PhD}$ thesis, University of Edinburgh, Department of Computer Science, December 1981. Also published as CST-15-81.

[Ong88a] C.-H.L. Ong. Fully abstract models of the lazy lambda calculus. In 29th Annual Symposium on Foundations of Computer Science, pages 368-376, White Plains, NY, October 1988. IEEE.

[Ong88b] C.-H.L. Ong. The Lazy Lambda Calculus: An Investigation into the Foundations of Functional Programming. PhD thesis, University of London, 1988. To appear. 
[Par91] A. Partridge. Dynamic Aspects of Distributed Graph Reduction. PhD thesis, Department of Electrical Engineering and Computer Science, University of Tasmania, 1991. In preparation.

[Pey87] S.L. Peyton Jones. The Implementation of Functional Programming Languages. Prentice-Hall International Series in Computer Science. Prentice-Hall International (UK) Ltd, London, 1987.

[Pey89] S.L. Peyton Jones. Parallel implementations of functional programming languages. The Computer Journal, 32(2):175-186, April 1989.

[Plo77] G. Plotkin. LCF considered as a programming language. Theoretical Computer Science, 5(3):223-256, 1977.

[Rea89] C. Reade. Elements of Functional Programming. International Computer Science Series. Addison-Wesley, 1989.

[SP82] M.B. Smyth and G.D. Plotkin. The category-theoretic solution of recursive domain equations. SIAM Journal on Computing, 11(4):761-783, November 1982.

[Tur86] D.A. Turner. An overview of Miranda. SIGPLAN Notices, December 1986.

[Wad71] C.P. Wadsworth. Semantics and Pragmatics of The Lambda Calculus. DPhil thesis, University of Oxford, 1971.

[Wad87] P.L. Wadler. Strictness analysis on non-flat domains (by abstract interpretation over finite domains). In S. Abramsky and C.L. Hankin, editors, Abstract Interpretation of Declarative Languages, chapter 12, pages 266-275. Ellis Horwood Ltd., Chichester, West Sussex, England, 1987.

[WH87] P. Wadler and R. J. M. Hughes. Projections for strictness analysis. In G. Kahn, editor, Proceedings of the Functional Programming Languages and Computer Architecture Conference, pages 385-407. Springer-Verlag LNCS 274, September 1987. 